\title{
On Identities of the Rogers-Ramanujan Type
}

ANDREW V. SILLS

asills@math.rutgers.edu

Department of Mathematics, Rutgers University, Hill Center-Busch Campus, Piscataway, NJ 08854

\begin{abstract}
A generalized Bailey pair, which contains several special cases considered by Bailey (Proc. London Math. Soc. (2), 50 (1949), 421-435), is derived and used to find a number of new Rogers-Ramanujan type identities. Consideration of associated $q$-difference equations points to a connection with a mild extension of Gordon's combinatorial generalization of the Rogers-Ramanujan identities (Amer. J. Math., 83 (1961), 393-399). This, in turn, allows the formulation of natural combinatorial interpretations of many of the identities in Slater's list (Proc. London Math. Soc. (2) 54 (1952), 147-167), as well as the new identities presented here. A list of 26 new double sum-product Rogers-Ramanujan type identities are included as an appendix.
\end{abstract}

Keywords: Rogers-Ramanujan identities, Bailey pairs, partitions

\section{Introduction}

\subsection{Overview}

We begin by recalling the famous Rogers-Ramanujan identities:

\section{The Rogers-Ramanujan Identities}

$$
\sum_{n=0}^{\infty} \frac{q^{n^{2}}}{(q ; q)_{n}}=\frac{\left(q^{2}, q^{3}, q^{5} ; q^{5}\right)_{\infty}}{(q ; q)_{\infty}},
$$

and

$$
\sum_{n=0}^{\infty} \frac{q^{n^{2}+n}}{(q ; q)_{n}}=\frac{\left(q, q^{4}, q^{5} ; q^{5}\right)_{\infty}}{(q ; q)_{\infty}}
$$

where

$$
\begin{aligned}
& (a ; q)_{m}=\prod_{j=0}^{m-1}\left(1-a q^{j}\right), \\
& (a ; q)_{\infty}=\prod_{j=0}^{\infty}\left(1-a q^{j}\right),
\end{aligned}
$$

and

$$
\left(a_{1}, a_{2}, \ldots, a_{r} ; q\right)_{s}=\left(a_{1} ; q\right)_{s}\left(a_{2} ; q\right)_{s} \ldots\left(a_{r} ; q\right)_{s},
$$

and throughout this paper we assume $|q|<1$ to ensure convergence.

The Rogers-Ramanujan identities are due to L. J. Rogers [20], and were rediscovered independently by S. Ramanujan [17] and I. Schur [23]. In the 1940's, W. N. Bailey undertook a careful study of Rogers' work, and greatly simplified it in a pair of papers ([8] and [9]). In these papers, Bailey was able to prove what he termed " $a$-generalizations" (i.e. formulae 
with a second variable $a$ in addition to $q$ ), of the Rogers-Ramanujan identities and a number of additional identities of similar type (some of which were due to Rogers and others of which were new at the time). Hereafter, $a$-generalizations of Rogers-Ramanujan type identities will be referred to simply as " $a$-RRT identities."

By considering a certain "parametrized Bailey pair," we will be naturally led to a variety of $a$-RRT identities, some of which were found by Bailey, and others of which appear to be new. Some examples of new $a$-RRT identities include

$$
\begin{gathered}
\sum_{n \geqq 0} \frac{a^{n} q^{n(n+1) / 2}(-1 ; q)_{n}}{\left(a q ; q^{2}\right)_{n}(q ; q)_{n}} \\
=\frac{(-a q ; q)_{\infty}}{(a q ; q)_{\infty}} \sum_{r \geqq 0} \frac{(-1)^{r} a^{3 r} q^{5 r^{2}}(-1 ; q)_{2 r}\left(1-a q^{4 r}\right)\left(a ; q^{2}\right)_{r}}{(1-a)\left(q^{2} ; q^{2}\right)_{r}(-a q ; q)_{2 r}}
\end{gathered}
$$

and

$$
\begin{gathered}
\sum_{n \geqq 0} \sum_{r \geqq 0} \frac{a^{n+r} q^{n^{2}+2 r^{2}}}{\left(a q ; q^{2}\right)_{n}\left(q^{2} ; q^{2}\right)_{r}(q ; q)_{n-r}} \\
=\frac{1}{(a q ; q)_{\infty}} \sum_{r \geqq 0} \frac{(-1)^{r} a^{4 r} q^{9 r^{2}-r}\left(1-a q^{4 r}\right)\left(a ; q^{2}\right)_{r}}{(1-a)\left(q^{2} ; q^{2}\right)_{r}}
\end{gathered}
$$

From the $a$-RRT identities, such as (3) and (4), we may easily deduce elegant RogersRamanujan type identities (in $q$ only); in these instances we obtain:

$$
\sum_{n \geqq 0} \frac{q^{n(n+1) / 2}(-1 ; q)_{n}}{\left(q ; q^{2}\right)_{n}(q ; q)_{n}}=\frac{\left(q^{5}, q^{5}, q^{10} ; q^{10}\right)_{\infty}(-q ; q)_{\infty}}{(q ; q)_{\infty}},
$$

which, surprisingly is not included in Slater's list [26], and

$$
\sum_{n \geqq 0} \sum_{r \geqq 0} \frac{q^{n^{2}+2 r^{2}}}{\left(q ; q^{2}\right)_{n}\left(q^{2} ; q^{2}\right)_{r}(q ; q)_{n-2 r}}=\frac{\left(q^{8}, q^{10}, q^{18} ; q^{18}\right)_{\infty}}{(q ; q)_{\infty}} .
$$

Remark. The referee pointed out that (3) follows from the $e, d \rightarrow \infty, c=-1$ case of [14, p. 68, (3.5.7)] and that Bailey actually had a generalization of (3), namely [9, p. 6 (6.3)], which makes it all the more remarkable that (5) did not appear in Slater's list. As we shall see later, (3) and (5) follow from the $(d, k)=(2,3)$ case of the parametrized Bailey pair, and (4) and (6) follow from the $(d, k)=(2,4)$ case.

Once we have an $a$-RRT identity in hand, we then study the $q$-difference equations related to the associated set of of identities. Observing the patterns which emerge in the $q$-difference equations associated with various sets of identities, one is led to consider the following mild extension of Basil Gordon's partition theorem:

Theorem 1.1 Let $A_{d, k, i}(n)$ denote the number of partitions of $n$ into parts $\not \equiv 0, \pm d i$ $(\bmod 2 d k+d)$. Let $B_{d, k, i}(n)$ denote the number of partitions of $n$ wherein 
- The integer $d$ appears as a part at most $i-1$ times,

- the total number of appearances of $d j$ and $d j+d$ (i.e. any two consecutive multiples of d) together is at most $k-1$, and

- nonmultiples of d may appear as parts without restriction.

Then for $1 \leqq i \leqq k, A_{d, k, i}(n)=B_{d, k, i}(n)$.

Remark. The case $d=1$ is Gordon's partition theorem [15].

As we shall see, special cases of Theorem 1.1 provide new combinatorial interpretations for various identities in Slater's list [26], as well as for the new analytic identities presented here.

For example, consider the Rogers mod 14 identities, which appear in Slater [26] as identities (59), (60), and (61) (see (29)-(31)). We shall see that these may be interpreted combinatorially as the $d=2, k=3$ case of Theorem 1.1:

Corollary 1.2 For $i=1,2,3$, the number of partitions of $n$ into parts wherein

- 2 appears as a part at most $i-1$ times,

- the total number of appearances of any two consecutive even numbers is at most 2 , and

- odd numbers may appear as parts without restriction,

equals the number of partitions of $n$ into parts not congruent to $0, \pm 2 i(\bmod 14)$.

Similarly, the combinatorial interpretation of (6) is

Corollary 1.3 The number of partitions of $n$ into parts wherein

- 2 appears as a part at most 3 times,

- the total number of appearances of any two consecutive even numbers is at most 3 , and

- odd numbers may appear as parts without restriction,

equals the number of partitions of $n$ into parts not congruent to $0, \pm 8(\bmod 18)$.

\subsection{Background}

The part of Bailey's results necessary for this current discussion may be briefly summarized as follows:

Definition 1. A pair of sequences $\left(\alpha_{n}(a, q), \beta_{n}(a, q)\right)$ is called a Bailey pair if for $n \geqq 0$,

$$
\beta_{n}(a, q)=\sum_{r=0}^{n} \frac{\alpha_{r}(a, q)}{(q ; q)_{n-r}(a q ; q)_{n+r}} .
$$


In [8] and [9], Bailey proved the fundamental result now known as "Bailey's Lemma" (see also [6, Chapter 3]):

Bailey's Lemma If $\left(\alpha_{r}(a, q), \beta_{j}(a, q)\right)$ form a Bailey pair, then

$$
\begin{gathered}
\frac{1}{\left(\frac{a q}{\rho_{1}} ; q\right)_{n}\left(\frac{a q}{\rho_{2}} ; q\right)_{n}} \sum_{j \geqq 0} \frac{\left(\rho_{1} ; q\right)_{j}\left(\rho_{2} ; q\right)_{j}\left(\frac{a q}{\rho_{1} \rho_{2}} ; q\right)_{n-j}}{(q ; q)_{n-j}}\left(\frac{a q}{\rho_{1} \rho_{2}}\right)^{j} \beta_{j}(a, q) \\
=\sum_{r=0}^{n} \frac{\left(\rho_{1} ; q\right)_{r}\left(\rho_{2} ; q\right)_{r}}{\left(\frac{a q}{\rho_{1}} ; q\right)_{r}\left(\frac{a q}{\rho_{2}} ; q\right)_{r}(q ; q)_{n-r}(a q ; q)_{n+r}}\left(\frac{a q}{\rho_{1} \rho_{2}}\right)^{r} \alpha_{r}(a, q) .
\end{gathered}
$$

An immediate consequence of Bailey's Lemma is the following important corollary:

Corollary 1.4 If $\left(\alpha_{m}(a, q), \beta_{j}(a, q)\right)$ form a Bailey pair, then

$$
\begin{gathered}
\sum_{j \geqq 0} a^{j} q^{j^{2}} \beta_{j}(a, q)=\frac{1}{(a q ; q)_{\infty}} \sum_{m=0}^{\infty} a^{m} q^{m^{2}} \alpha_{m}(a, q), \\
\sum_{j \geqq 0} a^{j} q^{j^{2}}\left(-q ; q^{2}\right)_{j} \beta_{j}\left(a, q^{2}\right)=\frac{\left(-a q ; q^{2}\right)_{\infty}}{\left(a q^{2} ; q^{2}\right)_{\infty}} \sum_{m=0}^{\infty} \frac{a^{m} q^{m^{2}}\left(-q ; q^{2}\right)_{m}}{\left(-a q ; q^{2}\right)_{m}} \alpha_{m}\left(a, q^{2}\right),
\end{gathered}
$$

and

$$
\sum_{j \geqq 0} a^{j} q^{j(j+1) / 2}(-1 ; q)_{j} \beta_{j}(a, q)=\frac{(-a q ; q)_{\infty}}{(a q ; q)_{\infty}} \sum_{m=0}^{\infty} \frac{a^{m} q^{m(m+1) / 2}(-1 ; q)_{m}}{(-a q ; q)_{m}} \alpha_{m}(a, q) .
$$

Proof: First, let $n, \rho_{1} \rightarrow \infty$ in (8). Then, to obtain (9), let $\rho_{2} \rightarrow \infty$; to obtain (11), set $\rho_{2}=-1$; and finally to obtain (10), set $\rho_{2}=-\sqrt{q}$, and then replace $q$ by $q^{2}$ throughout.

Thus the substitution of any Bailey pair $\left(\alpha_{n}(a, q), \beta_{n}(a, q)\right)$ into (9), (10), or (11) yields an $a$-RRT identity. Bailey did exactly this in [8] and [9]. Setting $a=1$ or $a=q$, one obtains traditional Rogers-Ramanujan type identities in the variable $q$ only. Bailey's student L.J. Slater [26] obtained a list of 130 Rogers-Ramanujan type identities (in $q$ only) in precisely this way. In $\S 2$, we study a general Bailey pair for which several special cases were considered by Bailey himself in [9]. Next, in $\S 3$, we derive $q$-difference equations for various sets of $a$-RRT identities, and consider their partition theoretic implications in $\S 4$. The narrative is concluded with some observations and open questions in $\S 5$. Finally, an appendix containing 26 new double sum-product Rogers-Ramanujan type identities is included. 


\section{A Parametrized Bailey Pair}

In [8] and [9], Bailey considered several Bailey pairs which are special cases of a more general Bailey pair involving additional parameters $d$ and $k$ :

Theorem 2.1 Let $\lambda=-\frac{3}{2} d^{2}+d k+\frac{1}{2} d, h=\left|\frac{2 \lambda}{d}\right|$, and $t=d+h+2$. Let

$$
\alpha_{d, k, m}(a, q):=\left\{\begin{array}{c}
\frac{(-1)^{r} a^{(k-d) r} q^{\left(d k-d^{2}+\frac{d}{2}\right) r^{2}-\frac{d}{2} r}\left(a q^{2 d} ; q^{2 d}\right)_{r}\left(a ; q^{d}\right)_{r}}{\left(a ; q^{2 d}\right)_{r}\left(q^{d} ; q^{d}\right)_{r}}, \\
\text { if } m=d r, \text { and } \\
0, \quad \text { otherwise },
\end{array}\right.
$$

and

$$
\beta_{d, k, m}(a, q):=\left\{\begin{array}{c}
\lim _{\tau \rightarrow 0} \frac{t+1}{W_{t}\left(a ; \nu_{1}, \ldots, \nu_{h}, \mu_{1}, \ldots, \mu_{d} ; q^{d} ; \tau^{h} a^{k-d} q^{n d}\right)} \\
(q, a q ; q)_{n} \\
\text { if } \lambda \geqq 0, \\
\lim _{\tau \rightarrow 0} \frac{t+1}{W_{t}\left(a ; \delta_{1}, \ldots, \delta_{h}, \mu_{1}, \ldots, \mu_{d} ; q^{d} ; \frac{a^{k-d} q^{n d}}{\tau^{h}}\right)} \\
(q, a q ; q)_{n} \\
\text { if } \lambda<0,
\end{array}\right.
$$

where $\nu_{j}=\frac{q^{\lambda / h}}{\tau}, \mu_{j}=q^{d-j-n}, \delta_{j}=\tau a q^{d-\lambda / h}$,

$$
{ }_{s+1} W_{s}\left(a_{1} ; a_{4}, a_{5}, \ldots, a_{s+1} ; q, z\right)={ }_{s+1} \phi_{s}\left[\begin{array}{c}
a_{1}, q a_{1}^{\frac{1}{2}},-q a_{1}^{\frac{1}{2}}, a_{4}, \ldots, a_{s+1} \\
a_{1}^{\frac{1}{2}},-a_{1}^{\frac{1}{2}}, \frac{q a_{1}}{a_{4}}, \ldots, \frac{q a_{1}}{a_{s+1}} ; q, z
\end{array}\right],
$$

and

$$
{ }_{s+1} \phi_{s}\left[\begin{array}{c}
a_{1}, a_{2}, \ldots, a_{s+1} \\
b_{1}, b_{2}, \ldots, b_{s}
\end{array} ; q, z\right]=\sum_{r=0}^{\infty} \frac{\left(a_{1}, a_{2}, \ldots, a_{s+1} ; q\right)_{r}}{\left(q, b_{1}, b_{2}, \ldots, b_{s} ; q\right)_{r}} z^{r} .
$$

Then $\left(\alpha_{d, k, m}(a, q), \beta_{d, k, n}(a, q)\right)$ form a Bailey pair.

Remark. The notation above is quite dense, and so a few words of clarification are perhaps in order. $\lambda$ represents the coëfficient of $r^{2}$ in the exponent of $q$ which arises when $\alpha_{d, k, m}(a, q)$ is inserted into the RHS of (7). $h$ is the number of rising $q$-factorials necessary to write $q^{\lambda r^{2}}$ as a limit as $\tau \rightarrow 0$ of a power of $\tau$ times the rising $q$ factorials in base $q^{d}$. For example, to write $q^{4 r^{2}}$ using base $q^{2}$, we find $h=4$ since

$$
q^{4 r^{2}}=\lim _{\tau \rightarrow 0} \tau^{4 r}\left(q / \tau ; q^{2}\right)_{r}^{4} .
$$

$t$ is the total number of denominator entries in the resulting very-well poised basic hypergeometric series. 
Proof of Theorem 2.1:

$$
\begin{aligned}
& \beta_{d, k, n}(a, q) \\
= & \sum_{m=0}^{n} \frac{1}{(q ; q)_{n-r}(a q ; q)_{n+r}} \alpha_{d, k, m}(a, q) \\
= & \frac{1}{(q ; q)_{n}(a q ; q)_{n}} \sum_{m=0}^{n} \frac{(-1)^{m} q^{n m+\frac{m}{2}-\frac{m^{2}}{2}}\left(q^{-n} ; q\right)_{m}}{\left(a q^{n+1} ; q\right)_{m}} \alpha_{d, k, m}(a, q) \\
= & \frac{1}{(q ; q)_{n}(a q ; q)_{n}} \sum_{r=0}^{\lfloor n / d\rfloor} \frac{(-1)^{d r} q^{n d r+\frac{d}{2} r-\frac{d^{2}}{2}} r^{2}\left(q^{-n} ; q\right)_{d r}}{\left(a q^{n+1} ; q\right)_{d r}} \alpha_{d, k, d r}(a, q) \\
= & \sum_{r=0}^{\lfloor n / d\rfloor} \frac{(-1)^{(d+1) r} a^{(k-d) r} q^{\lambda r^{2}+n d r}\left(a ; q^{d}\right)_{r}\left(a q^{2 d} ; q^{2 d}\right)_{r}\left(q^{-n} ; q\right)_{d r}}{(q ; q)_{n}(a q ; q)_{n}\left(q^{d} ; q^{d}\right)_{r}\left(a ; q^{2 d}\right)_{r}\left(a q^{n+1} ; q\right)_{d r}}
\end{aligned}
$$

If $\lambda \geqq 0$, this last expression

$$
\begin{gathered}
=\frac{1}{(q ; q)_{n}(a q ; q)_{n}} \lim _{\tau \rightarrow 0} \sum_{r \geqq 0}\left\{\frac{\left(a, q^{d} \sqrt{a},-q^{d} \sqrt{a} ; q^{d}\right)_{r}\left(q^{\lambda / h} / \tau ; q^{d}\right)_{r}^{h}}{\left(q^{d}, \sqrt{a},-\sqrt{a} ; q^{d}\right)_{r}\left(\tau a q^{d-\lambda / h} ; q^{d}\right)_{r}^{h}}\right. \\
\left.\times \frac{\left(q^{d-1-n}, q^{d-2-n}, \ldots, q^{-n} ; q^{d}\right)_{r}}{\left(a q^{n+1}, a q^{n+2}, \ldots, a q^{n+d} ; q^{d}\right)_{r}} \tau^{h r} a^{(k-d) r} q^{n d r}\right\},
\end{gathered}
$$

while if $\lambda<0$, we instead place $q^{-\lambda r^{2}}$ in the denominator:

$$
\begin{gathered}
=\frac{1}{(q ; q)_{n}(a q ; q)_{n}} \lim _{\tau \rightarrow 0} \sum_{r \geqq 0}\left\{\frac{\left(a, q^{d} \sqrt{a},-q^{d} \sqrt{a} ; q^{d}\right)_{r}\left(\tau a q^{d-\lambda / h} ; q^{d}\right)_{r}^{h}}{\left(q^{d}, \sqrt{a},-\sqrt{a} ; q^{d}\right)_{r}\left(q^{\lambda / h} / \tau ; q^{d}\right)_{r}^{h}}\right. \\
\left.\times \frac{\left(q^{d-1-n}, q^{d-2-n}, \ldots, q^{-n} ; q^{d}\right)_{r}}{\left(a q^{n+1}, a q^{n+2}, \ldots, a q^{n+d} ; q^{d}\right)_{r}} \tau^{h r} a^{(k-d) r} q^{n d r}\right\} .
\end{gathered}
$$

The goal is to find Bailey pairs which will give rise to attractive identities. Bailey himself considered the special cases $\alpha_{d, k, m}(a, q)$ for $(d, k)=(1,2),(2,2),(2,3)$, and $(3,4)[9$, p. 5-6, eqns. (i), (iv) with $f=0$, (iv) with $f \rightarrow \infty$, and (v) respectively]. Each of these four $(d, k)$ sets is particularly nice, as the resulting expression for $\alpha_{d, k, r}(a, q)$, when substituted into (7), is a finite product times a ${ }_{6} W_{5}$ on base $q^{d}$, which is summable by Jackson's theorem [14, p. 238, eqn. (II.20)]. Thus, $\beta_{d, k, n}(a, q)$ reduces to a finite product, and upon substituting it into (9), the left hand side of the resulting $a$-RRT identity will be a single-fold sum.

In this way, upon letting $a \rightarrow 1$, we may derive the first Rogers-Ramanujan identity (1) from $(d, k)=(1,2)$, a Rogers' mod 10 identity (25) from $(d, k)=(2,2)$, a Rogers mod 14 identity (31) from $(d, k)=(2,3)$, and a Bailey-Dyson mod 27 identity (35) from $(d, k)=(3,4)$. It was not mentioned by Bailey, but Euler's pentagonal number theorem [2, p. 11, Cor. 1.7] arises from the case $(d, k)=(1,1)$. Similarly, by substituting the Bailey 
pairs into (10) and (11), and then letting $a \rightarrow 1$, other identities from Slater's list may be derived. One case that both Bailey and Slater seem to have missed is the substitution of $(d, k)=(2,3)$ into (11), which immediately yields (3) and then (5) when $a=1$.

Note that, in fact, $d=1$ corresponds to the "unit Bailey chain" [5]. Substituting the Bailey pairs corresponding to the $d=1$ cases into (9) yields cases of Andrews' analytic generalization of the Rogers-Ramanujan identities for odd moduli [3]; see (36).

Thus to search for new identities, we need to consider $d>1$. Also, in order to find $\beta_{d, k, n}$ 's with relatively simple forms, $d+h$ should be kept as small as possible since $\beta_{n}$ is a finite product times a ${ }_{d+h+3} W_{d+h+2}$, and the higher one looks in the hypergeometric hierarchy, the more complicated things become. It appears that Bailey considered all cases where $d+h=3$, and thus all of the summable ${ }_{6} W_{5}$ 's. The next best situation is where $d+h=5$, which corresponds to a ${ }_{8} W_{7}$ that can be transformed by Watson's $q$-analog of Whipple's Theorem [14, p. 242, eqn. (III.17)]:

Consider the case $(d, k)=(2,4)$ :

$$
\begin{aligned}
& \beta_{2,4, n}(a, q) \\
& =\frac{1}{(q ; q)_{n}(a q ; q)_{n}} \sum_{r=0}^{\lfloor n / 2\rfloor} \frac{(-1)^{r} a^{2 r} q^{3 r^{2}+2 n r}\left(a ; q^{2}\right)_{r}\left(a q^{4} ; q^{4}\right)_{r}\left(q^{-n} ; q\right)_{2 r}}{\left(q^{2} ; q^{2}\right)_{r}\left(a ; q^{4}\right)_{r}\left(a q^{n+1} ; q\right)_{2 r}} \\
& =\frac{1}{(q ; q)_{n}(a q ; q)_{n}} \\
& \times \lim _{\tau \rightarrow 0} 8 \phi_{7}\left[\begin{array}{c}
a, q^{2} \sqrt{a},-q^{2} \sqrt{a}, \frac{q}{\tau}, \frac{q}{\tau}, \frac{q}{\tau}, q^{1-n}, q^{-n} \\
\sqrt{a},-\sqrt{a}, \tau a q, \tau a q, \tau a q, a q^{n+1}, a q^{n+2}
\end{array} ; q^{2}, \tau^{3} a^{2} q^{2 n}\right] \\
& =\frac{1}{(q ; q)_{n}(a q ; q)_{n}} \lim _{\tau \rightarrow 0} \frac{\left(a q^{2}, \tau a q^{n+1}, \tau a q^{n}, a q^{2 n+1} ; q^{2}\right)_{\infty}}{\left(a q \tau, a q^{n+2}, a q^{n+1}, \tau a q^{2 n} ; q^{2}\right)_{\infty}} \\
& \times_{4} \phi_{3}\left[\begin{array}{c}
\tau^{2} a, \frac{q}{\tau}, q^{-n}, q^{1-n} \\
\tau a q, \tau a q, \frac{q^{2-2 n}}{a \tau}
\end{array} ; q^{2}, q^{2}\right] \\
& =\frac{\left(a q^{2}, a q^{2 n+1} ; q^{2}\right)_{\infty}}{(q, a q ; q)_{n}\left(a q^{n+1} ; q\right)_{\infty}} \sum_{r \geqq 0} \frac{\left(q^{-n} ; q\right)_{2 r}}{\left(q^{2} ; q^{2}\right)_{r}} a^{r} q^{2 n r+r} \\
& =\frac{1}{\left(a q ; q^{2}\right)_{n}} \sum_{r \geqq 0} \frac{a^{r} q^{2 r^{2}}}{\left(q^{2} ; q^{2}\right)_{r}(q ; q)_{n-2 r}} \text {. }
\end{aligned}
$$

Analogous calculations allow us to find

$$
\begin{aligned}
& \beta_{2,1, n}(a, q)=\frac{q^{\left(\begin{array}{c}
n \\
2
\end{array}\right)}}{\left(a q ; q^{2}\right)_{n}} \sum_{r \geqq 0} \frac{(-1)^{r} a^{-r} q^{r^{2}-2 n r}}{\left(q^{2} ; q^{2}\right)_{r}(q ; q)_{n-2 r}} \\
& \beta_{3,3, n}(a, q)=\frac{1}{(a ; q)_{2 n}} \sum_{r \geqq 0} \frac{(-1)^{r} q^{\frac{3}{2} r^{2}-\frac{3}{2} r}\left(a ; q^{3}\right)_{n-r}}{\left(q^{3} ; q^{3}\right)_{r}(q ; q)_{n-3 r}} \\
& \beta_{3,5, n}(a, q)=\frac{1}{(a ; q)_{2 n}} \sum_{r \geqq 0} \frac{a^{r} q^{3 r^{2}}\left(a ; q^{3}\right)_{n-r}}{\left(q^{3} ; q^{3}\right)_{r}(q ; q)_{n-3 r}} .
\end{aligned}
$$


Once $d>3$, even if $d+h=5$, Watson's $q$-Whipple transformation [14, p. 242, eqn. (III.17)] is not applicable as the resulting ${ }_{4} \phi_{3}$ does not terminate. In this case, we must use the more general transformation [14, p. 246, eqn. (III.36)]. Let us now consider such a situation:

$$
\begin{aligned}
& \beta_{4,6, n}(a, q) \\
& =\frac{1}{(q, a q ; q)_{n}} \lim _{\tau \rightarrow 0}{ }_{8} W_{7}\left[a ; \frac{q^{2}}{\tau^{2}}, q^{1-n}, q^{3-n}, q^{-n}, q^{2-n} ; q^{4} ; \tau^{2} a^{2} q^{4 n}\right] \\
& =\lim _{\tau \rightarrow 0} \frac{\left(a q^{2}, a q^{n-1} \tau, a q^{2 n+1},-a q^{2 n+1}, \tau^{\frac{1}{2}} a q^{n+1},-\tau^{\frac{1}{2}} a q^{n+1}\right)_{\infty}}{(q, a q ; q)_{n}\left(a \tau, a q^{n+1}, a q^{n+2},-a q^{n+2}, \tau a q^{2 n},-\tau a q^{2 n} ; q^{2}\right)_{\infty}} \\
& \times_{8} \phi_{7}\left[\begin{array}{c}
-a q^{n}, i a^{\frac{1}{2}} q^{2+\frac{n}{2}},-i a^{\frac{1}{2}} q^{2+\frac{n}{2}}, q^{1-n}, \frac{q}{\tau},-\frac{q}{\tau}, a^{\frac{1}{2}} q^{1+n},-a^{\frac{1}{2}} q^{1+n} \\
i a^{\frac{1}{2}} q^{\frac{n}{2}},-i a^{\frac{1}{2}} q^{\frac{n}{2}},-a q^{2 n+1},-\tau a q^{n+1}, \tau a q^{n+1},-q a^{\frac{1}{2}}, q a^{\frac{1}{2}}
\end{array} ; q^{2}, a q^{n-1}\right] \\
& \text { (by [14, p. 70, eqn. (3.5.10)]) } \\
& =\lim _{\tau \rightarrow 0} \frac{\left(a q^{2}, \tau^{2} a q^{n-1}, a q^{2 n+1},-a q^{2 n+1}, \tau a q^{n+1}-\tau a q^{n+1} ; q^{2}\right)_{\infty}}{(q, a q ; q)_{n}\left(\tau^{2} a, a q^{n+1}, a q^{n+2},-a q^{n+2}, \tau a q^{2 n},-\tau a q^{2 n} ; q^{2}\right)_{\infty}} \\
& \times\left\{\frac{\left(-a q^{n+2}, q^{-n},-q^{n} a^{\frac{1}{2}}, q^{n} a^{\frac{1}{2}} ; q^{2}\right)_{\infty}}{\left(-q a^{\frac{1}{2}}, q a^{\frac{1}{2}},-a q^{2 n+1}, \frac{1}{q} ; q^{2}\right)_{\infty}}\right.
\end{aligned}
$$

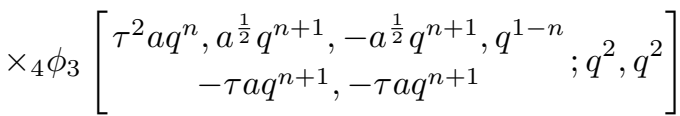

$$
\begin{aligned}
& +\frac{\left(-a q^{n+2}, \tau^{2} a q^{n}, a^{\frac{1}{2}} q^{n+1},-a^{\frac{1}{2}} q^{n+1},-\tau a q^{n}, \tau a q^{n} ; q^{2}\right)_{\infty}}{\left(-\tau a q^{n+1}, \tau a q^{n+1},-q a^{\frac{1}{2}}, q a^{\frac{1}{2}},-a q^{2 n+1}, a q^{2 n+1}, \tau^{2} a q^{n-1}, q ; q^{2}\right)_{\infty}} \\
& \left.\times_{4} \phi_{3}\left[\begin{array}{c}
q^{-n},-q^{n} a^{\frac{1}{2}}, q^{n} a^{\frac{1}{2}}, \tau^{2} a q^{n-1} ; q^{2}, q^{2} \\
-\tau a q^{n}, \tau a q^{n}, q
\end{array}\right]\right\} \\
& \text { (by [14, p. 246, eqn. (III.36)]) } \\
& =\frac{1}{(q ; q)_{n}\left(a q ; q^{2}\right)_{n}\left(a q^{2} ; q^{4}\right)_{\infty}\left(q ; q^{2}\right)_{\infty}} \\
& \times\left\{-\left(q^{-n} ; q^{2}\right)_{\infty}\left(a q^{2 n} ; q^{4}\right)_{\infty} \sum_{r \geqq 0} \frac{\left(a q^{2 n+2} ; q^{4}\right)_{r}\left(q^{1-n} ; q^{2}\right)_{r} q^{2 r+1}}{(q ; q)_{2 r+1}}\right. \\
& \left.+\left(q^{1-n} ; q^{2}\right)_{\infty}\left(a q^{2 n+2} ; q^{4}\right)_{\infty} \sum_{r \geqq 0} \frac{\left(a q^{2 n} ; q^{4}\right)_{r}\left(q^{-n} ; q^{2}\right)_{r} q^{2 r}}{(q ; q)_{2 r}}\right\}
\end{aligned}
$$

Notice that the first term vanishes for $n$ even and the second for $n$ odd. Thus we conclude

$$
\beta_{4,6,2 m}(a, q)=\sum_{r \geqq 0} \frac{(-1)^{m+r} q^{r^{2}-m^{2}+r-2 m r}\left(a ; q^{4}\right)_{m+r}}{(a ; q)_{4 m}(q ; q)_{2 r}\left(q^{2} ; q^{2}\right)_{m-r}}
$$


and

$$
\beta_{4,6,2 m+1}(a, q)=\sum_{r \geqq 0} \frac{(-1)^{m+r} q^{r^{2}-m^{2}+r-2 m-2 m r}\left(a ; q^{4}\right)_{m+r+1}}{(a ; q)_{4 m+2}(q ; q)_{2 r+1}\left(q^{2} ; q^{2}\right)_{m-r}} .
$$

\section{3. q-difference equations}

For each of the Bailey pairs derived in $\S 2$, we are able to obtain one $a$-RRT identity from each of (9), (10), and (11). However, in general there are a set of $k$ identities associated with a given $(d, k)$. We will use $q$-difference equations to establish complete sets of $k$ identities for various $(d, k)$ considered in $\S 2$, as well as those $(d, k)$ considered by Bailey [9].

\subsection{Expressions for the right hand sides and their $\boldsymbol{q}$-difference equations}

Definition 2. For $k \geqq 1$, and $1 \leqq i \leqq k$,

$$
\begin{gathered}
Q_{d, k, i}(a):=Q_{d, k, i}(a, q) \\
:=\frac{1}{(a q ; q)_{\infty}} \sum_{n \geqq 0} \frac{(-1)^{n} a^{k n} q^{\left(d k+\frac{d}{2}\right) n^{2}+\left(k-i+\frac{1}{2}\right) d n}\left(1-a^{i} q^{(2 n+1) d i}\right)\left(a q^{d} ; q^{d}\right)_{n}}{\left(q^{d} ; q^{d}\right)_{n}} .
\end{gathered}
$$

Theorem 3.1 The following q-difference equations are valid:

$$
Q_{d, k, 1}(a)=\frac{1}{(a q ; q)_{d-1}} Q_{d, k, k}\left(a q^{d}\right)
$$

and for $2 \leqq i \leqq k$,

$$
Q_{d, k, i}(a)=Q_{d, k, i-1}(a)+\frac{a^{i-1} q^{(i-1) d}}{(a q ; q)_{d-1}} Q_{d, k, k-i+1}\left(a q^{d}\right) .
$$

Before proving Theorem 3.1, we need the following lemma:

\section{Lemma 3.2}

$$
Q_{d, k, k}(a)=\frac{1}{(a q ; q)_{\infty}} \sum_{n \geqq 0} \frac{(-1)^{n} a^{k n} q^{\left(d k+\frac{d}{2}\right) n^{2}-\frac{d}{2} n}\left(1-a q^{2 d n}\right)\left(a ; q^{d}\right)_{n}}{(1-a)\left(q^{d} ; q^{d}\right)_{n}}
$$


Proof:

$$
\begin{aligned}
& \sum_{n \geqq 0} \frac{(-1)^{n} a^{k n} q^{\left(d k+\frac{d}{2}\right) n^{2}-\frac{d}{2} n}\left(1-a q^{2 d n}\right)\left(a ; q^{d}\right)_{n}}{(1-a)\left(q^{d} ; q^{d}\right)_{n}} \\
= & \sum_{n \geqq 0} \frac{(-1)^{n} a^{k n} q^{\left(d k+\frac{d}{2}\right) n^{2}-\frac{d}{2} n}\left(a ; q^{d}\right)_{n}}{(1-a)\left(q^{d} ; q^{d}\right)_{n}}\left\{q^{d n}\left(1-a q^{d n}\right)+\left(1-q^{d n}\right)\right\} \\
= & \sum_{n \geqq 0} \frac{(-1)^{n} a^{k n} q^{\left(d k+\frac{d}{2}\right) n^{2}+\frac{d}{2} n}\left(a ; q^{d}\right)_{n+1}}{(1-a)\left(q^{d} ; q^{d}\right)_{n}} \\
& +\sum_{n \geqq 1} \frac{(-1)^{n} a^{k n} q^{\left(d k+\frac{d}{2}\right) n^{2}-\frac{d}{2} n}\left(a q^{d} ; q^{d}\right)_{n-1}}{\left(q^{d} ; q^{d}\right)_{n-1}} \\
= & \sum_{n \geqq 0} \frac{(-1)^{n} a^{k n} q^{\left(d k+\frac{d}{2}\right) n^{2}+\frac{d}{2} n}\left(a q^{d} ; q^{d}\right)_{n}}{\left(q^{d} ; q^{d}\right)_{n}} \\
& -\sum_{n \geqq 0} \frac{(-1)^{n} a^{k n+k} q^{\left(d k+\frac{d}{2}\right) n^{2}+\left(2 d k+\frac{d}{2}\right) n+d k}\left(a q^{d} ; q^{d}\right)_{n}}{\left(q^{d} ; q^{d}\right)_{n}} \\
= & \sum_{n \geqq 0} \frac{(-1)^{n} a^{k n} q^{\left(d k+\frac{d}{2}\right) n^{2}+\left(\frac{d}{2}\right) n}\left(a q^{d} ; q^{d}\right)_{n}\left(1-a^{k} q^{(2 n+1) d k}\right)}{\left(q^{d} ; q^{d}\right)_{n}} \\
= & (a q ; q)_{\infty} Q_{d, k, k}(a) .
\end{aligned}
$$

Proof: Proof of (19)

$$
\begin{aligned}
& \frac{1}{(a q ; q)_{d-1}} Q_{d, k, k}\left(a q^{d}\right) \\
= & \frac{1}{(a q ; q)_{d-1}\left(a q^{d+1} ; q\right)_{\infty}} \\
& \quad \times \sum_{n \geqq 0} \frac{(-1)^{n} a^{k n} q^{\left(d k+\frac{d}{2}\right) n^{2}+\left(d k-\frac{d}{2}\right) n}\left(1-a q^{(2 n+1) d}\right)\left(a q^{d} ; q^{d}\right)_{n}}{\left(1-a q^{d}\right)\left(q^{d} ; q^{d}\right)_{n}} \\
= & \frac{1}{(a q ; q)_{\infty}} \sum_{n \geqq 0} \frac{(-1)^{n} a^{k n} q^{\left(d k+\frac{d}{2}\right) n^{2}+\left(d k-\frac{d}{2}\right) n}\left(1-a q^{(2 n+1) d}\right)\left(a q^{d} ; q^{d}\right)_{n}}{\left(q^{d} ; q^{d}\right)_{n}} \\
= & Q_{d, k, 1}(a) .
\end{aligned}
$$

Proof: Proof of (20)

$$
Q_{d, k, i}(a)-Q_{d, k, i-1}(a)
$$




$$
\begin{aligned}
& =\frac{1}{(a q ; q)_{\infty}} \sum_{n=0}^{\infty} \frac{(-1)^{n} a^{k n} q^{\left(k+\frac{1}{2}\right) d n^{2}+\left(k+\frac{1}{2}\right) d n}\left(a q^{d} ; q^{d}\right)_{n}}{\left(q^{d} ; q^{d}\right)_{n}} \\
& \quad \times\left(q^{-i d n}\left(1-a^{i} q^{(2 n+1) d i}\right)-q^{d n(1-i)}\left(1-a^{i-1} q^{(2 n+1) d(i-1)}\right)\right) \\
& =\frac{1}{(a q ; q)_{\infty}} \sum_{n=0}^{\infty} \frac{(-1)^{n} a^{k n} q^{\left(k+\frac{1}{2}\right) d n^{2}+\left(k+\frac{1}{2}\right) d n}\left(a q^{d} ; q^{d}\right)_{n}}{\left(q^{d} ; q^{d}\right)_{n}} \\
& \quad \times\left(q^{-i d n}\left(1-q^{d n}\right)+a^{i-1} q^{d(n+1)(i-1)}\left(1-a q^{d(n+1)}\right)\right) \\
& =\frac{1}{(a q ; q)_{\infty}}\left(\sum_{n=1}^{\infty} \frac{(-1)^{n} a^{k n} q^{\left(k+\frac{1}{2}\right) d n^{2}+\left(k-i+\frac{1}{2}\right) d n}\left(a q^{d} ; q^{d}\right)_{n}}{\left(q^{d} ; q^{d}\right)_{n-1}}\right. \\
& \left.\quad+\sum_{n=0}^{\infty} \frac{(-1)^{n} a^{k n+i-1} q^{\left(k+\frac{1}{2}\right) d n^{2}+\left(k+\frac{1}{2}\right) d n+d(n+1)(i-1)}\left(a q^{d} ; q^{d}\right)_{n+1}}{\left(q^{d} ; q^{d}\right)_{n}}\right) \\
& =\frac{1}{(a q ; q)_{\infty}}\left(-\sum_{n=0}^{\infty} \frac{(-1)^{n} a^{k n+k} q^{\left(k+\frac{1}{2}\right) d n^{2}+\left(3 k-i+\frac{3}{2}\right) d n+d(2 k-i+1)}\left(a q^{d} ; q^{d}\right)_{n+1}}{\left(q^{d} ; q^{d}\right)_{n}}\right. \\
& \left.\quad+\sum_{n=0}^{\infty} \frac{(-1)^{n} a^{k n+i-1} q^{\left(k+\frac{1}{2}\right) d n^{2}+\left(k+i-\frac{1}{2}\right) d n+d(i-1)}\left(a q^{d} ; q^{d}\right)_{n+1}}{\left(q^{d} ; q^{d}\right)_{n}}\right) \\
& =\frac{a^{i-1} q^{d(i-1)}\left(1-a q^{d}\right)}{(a q ; q)_{d}\left(a q^{d+1} ; q\right)_{\infty}}\left(\sum_{n=0}^{\infty} \frac{(-1)^{n} a^{k n} q^{\left(k+\frac{1}{2}\right) d n^{2}+\left(k+i-\frac{1}{2}\right) d n}\left(a q^{2 d} ; q^{d}\right)_{n}}{\left(q^{d} ; q^{d}\right)_{n}}\right. \\
& \quad-\sum_{n=0}^{\infty} \frac{(-1)^{n} a^{k n+k-i+1} q^{\left(k+\frac{1}{2}\right) d n^{2}+\left(3 k-i+\frac{3}{2}\right) d n+2 d(k-i+1)}\left(a q^{2 d} ; q^{d}\right)_{n}}{\left(q^{d} ; q^{d}\right)_{n}} \\
& =\frac{a^{i-1} q^{d(i-1)}}{(a q ; q)_{d-1}\left(a q^{d+1} ; q\right)_{\infty}} \sum_{n=0}^{\infty} \frac{(-1)^{n} a^{k n} q^{\left(k+\frac{1}{2}\right) d n^{2}+\left(k+i-\frac{1}{2}\right) d n}\left(a q^{2 d} ; q^{d}\right)_{n}}{\left(a q ; q q_{d-1} ; q^{d}\right)_{n}} \\
& \times\left(1-a^{k-i+1} q^{2 d(n+1)(k-i+1)}\right)
\end{aligned}
$$

Remark. Note that for $1 \leqq i \leqq k, Q_{d, k, i}(0, q)=1$ which, together with (19) and (20) uniquely determine $Q_{d, k, i}(a, q)$ as a power series in $a$ and $q$. In $\S 3.2$, we will show that certain functions $F_{d, k, i}(a, q)$ satisfy the same recurrence and initial conditions as the $Q_{d, k, i}(a, q)$ for various values of $d$ and $k$, thus yielding collections of $a$-RRT identities. Then, in $\S 4$, we will see that generating functions for certain classes of partitions satisfy those same recurrences and initial conditions, thus providing partition identities.

Rogers-Ramanujan type identities (in $q$ only) are perhaps more aesthetically pleasing than their $a$-RRT counterparts because their right hand sides are expressible as infinite products. Accordingly, we prove the following proposition for later use. 


\section{Proposition 3.3}

$$
Q_{d, k, i}(1)=\frac{\left(q^{i d}, q^{(2 k-i+1) d}, q^{(2 k+1) d} ; q^{(2 k+1) d}\right)_{\infty}}{(q ; q)_{\infty}}
$$

Proof:

$$
\begin{aligned}
& (q ; q)_{\infty} Q_{d, k, i}(1) \\
= & \sum_{n \geqq 0}(-1)^{n} q^{\left(d k+\frac{d}{2}\right) n^{2}+\left(k-i+\frac{1}{2}\right) d n}\left(1-q^{(2 n+1) d i}\right) \\
= & \sum_{n \geqq 0}(-1)^{n} q^{\left(d k+\frac{d}{2}\right) n^{2}+\left(d k-d i+\frac{d}{2}\right) n}-(-1)^{n} q^{\left(d k+\frac{d}{2}\right) n^{2}+\left(d k+d i+\frac{d}{2}\right) n+d i} \\
= & \sum_{n \geqq 0}(-1)^{n} q^{\left(d k+\frac{d}{2}\right) n^{2}-\left(d i-d k-\frac{d}{2}\right) n}+\sum_{n=1}^{\infty}(-1)^{n} q^{\left(d k+\frac{d}{2}\right) n^{2}+\left(d i-d k-\frac{d}{2}\right) n} \\
= & \sum_{n=-\infty}^{\infty}(-1)^{n} q^{\left(d k+\frac{d}{2}\right) n^{2}-\left(d k-d i+\frac{d}{2}\right) n} \\
= & \left(q^{d i}, q^{2 d k-d i+d}, q^{2 d k+d} ; q^{2 d k+d}\right)_{\infty}
\end{aligned}
$$

(by Jacobi's triple product identity [2, p. 21, Theorem 2.8])

\subsection{Expressions for the left hand sides and their $\mathbf{q}$-difference equations}

We now work out the $q$-difference equations associated with the left hand sides of various $a$-RRT identities.

3.2.1. The case $(d, k)=(2,2)$

Definition 3.

$$
\begin{aligned}
F_{2,2,1}(a) & :=F_{2,2,1}(a, q):=\sum_{n=0}^{\infty} \frac{a^{n} q^{\frac{3}{2} n^{2}+\frac{3}{2} n}}{\left(a q ; q^{2}\right)_{n+1}(q ; q)_{n}} . \\
F_{2,2,2}(a) & :=F_{2,2,2}(a, q):=\sum_{n=0}^{\infty} \frac{a^{n} q^{\frac{3}{2} n^{2}-\frac{1}{2} n}}{\left(a q ; q^{2}\right)_{n}(q ; q)_{n}} \\
F_{2,2,2}^{*}(a) & :=F_{2,2,2}^{*}(a, q):=\sum_{n=0}^{\infty} \frac{a^{n} q^{\frac{3}{2} n^{2}+\frac{1}{2} n}}{\left(a q ; q^{2}\right)_{n+1}(q ; q)_{n}}
\end{aligned}
$$

Lemma $3.4 F_{2,2,2}(a)=F_{2,2,2}^{*}(a)$. 
Proof:

$$
\begin{aligned}
& F_{2,2,2}(a)-F_{2,2,2}^{*}(a) \\
= & \sum_{n=0}^{\infty} \frac{a^{n} q^{\frac{3}{2}} n^{2}-\frac{1}{2} n}{\left(a q ; q^{2}\right)_{n+1}(q ; q)_{n}}\left(\left(1-a q^{2 n+1}\right)-q^{n}\right) \\
= & \sum_{n=0}^{\infty} \frac{a^{n} q^{\frac{3}{2}} n^{2}-\frac{1}{2} n}{\left(a q ; q^{2}\right)_{n+1}(q ; q)_{n}}\left(\left(1-a q^{2 n+1}\right)\left(1-q^{n}\right)-a q^{3 n+1}\right) \\
= & \sum_{n=1}^{\infty} \frac{a^{n} q^{\frac{3}{2}} n^{2}-\frac{1}{2} n}{\left(a q ; q^{2}\right)_{n}(q ; q)_{n-1}}-\sum_{n=0}^{\infty} \frac{a^{n+1} q^{\frac{3}{2} n^{2}+\frac{5}{2} n+1}}{\left(a q ; q^{2}\right)_{n+1}(q ; q)_{n}} \\
= & 0
\end{aligned}
$$

Lemma 3.5 The $F_{2,2, i}(a, q)$ satisfy the following $q$-difference equations:

$$
\begin{gathered}
F_{2,2,1}(a)=\frac{1}{1-a q} F_{2,2,2}\left(a q^{2}\right) \\
F_{2,2,2}(a)=F_{2,2,1}(a)+\frac{a q^{2}}{1-a q} F_{2,2,1}\left(a q^{2}\right)
\end{gathered}
$$

which, together with $F_{2,2, i}(0)=1$ for $i=1,2$, uniquely determine $F_{2,2, i}(a)$ as a double power series in a and $q$.

Proof: By inspection, we see

$$
\frac{1}{1-a q} F_{2,2,2}^{*}\left(a q^{2}\right)=\frac{1}{1-a q} F_{2,2,2}\left(a q^{2}\right)=F_{2,2,1}(a),
$$

and so (22) is established. Next,

$$
\begin{aligned}
F_{2,2,2}^{*}(a)-F_{2,2,1}(a) & =\sum_{n=1}^{\infty} \frac{a^{n} q^{\frac{3}{2} n^{2}+\frac{1}{2} n}\left(1-q^{n}\right)}{\left(a q ; q^{2}\right)_{n+1}(q ; q)_{n}} \\
& =\sum_{n=0}^{\infty} \frac{a^{n+1} q^{\frac{3}{2} n^{2}+\frac{7}{2} n+2}}{\left(a q ; q^{2}\right)_{n+2}(q ; q)_{n}} \\
& =\frac{a q^{2}}{1-a q} F_{2,2,1}\left(a q^{2}\right),
\end{aligned}
$$

which verifies (23).

Thus, by combining Lemma 3.5 with Theorem 3.1, we have established the following theorem:

Theorem 3.6 For $i=1,2$,

$$
F_{2,2, i}(a)=Q_{2,2, i}(a) .
$$

Setting $a=1$ and employing Proposition 3.3, we obtain two identities of Rogers [20], which appear as (44) and (46) on Slater's list [26]: 


\section{Corollary 3.7}

$$
\begin{gathered}
\sum_{n=0}^{\infty} \frac{q^{\frac{3}{2} n^{2}+\frac{3}{2} n}}{\left(q ; q^{2}\right)_{n+1}(q ; q)_{n}}=\frac{\left(q^{2}, q^{8}, q^{10} ; q^{10}\right)_{\infty}}{(q ; q)_{\infty}} \\
\sum_{n=0}^{\infty} \frac{q^{\frac{3}{2} n^{2}-\frac{1}{2} n}}{\left(q ; q^{2}\right)_{n}(q ; q)_{n}}=\sum_{n=0}^{\infty} \frac{q^{\frac{3}{2} n^{2}+\frac{1}{2} n}}{\left(q ; q^{2}\right)_{n+1}(q ; q)_{n}}=\frac{\left(q^{4}, q^{6}, q^{10} ; q^{10}\right)_{\infty}}{(q ; q)_{\infty}} .
\end{gathered}
$$

3.2.2. The case $(d, k)=(2,3)$

\section{Definition 4.}

$$
\begin{aligned}
F_{2,3,1}(a) & :=F_{2,3,1}(a, q):=\sum_{n=0}^{\infty} \frac{a^{n} q^{n^{2}+2 n}}{\left(a q ; q^{2}\right)_{n+1}(q ; q)_{n}} \\
F_{2,3,2}(a) & :=F_{2,3,2}(a, q):=\sum_{n=0}^{\infty} \frac{a^{n} q^{n^{2}+n}}{\left(a q ; q^{2}\right)_{n+1}(q ; q)_{n}} \\
F_{2,3,3}(a) & :=F_{2,3,3}(a, q):=\sum_{n=0}^{\infty} \frac{a^{n} q^{n^{2}}}{\left(a q ; q^{2}\right)_{n}(q ; q)_{n}}
\end{aligned}
$$

Lemma 3.8 The $F_{2,3, i}(a, q)$ satisfy the following q-difference equations:

$$
\begin{gathered}
F_{2,3,1}(a)=\frac{1}{1-a q} F_{2,3,3}\left(a q^{2}\right) \\
F_{2,3,2}(a)=F_{2,3,1}(a)+\frac{a q^{2}}{1-a q} F_{2,3,2}\left(a q^{2}\right) \\
F_{2,3,3}(a)=F_{2,3,2}(a)+\frac{a^{2} q^{4}}{1-a q} F_{2,3,1}\left(a q^{2}\right),
\end{gathered}
$$

which, together with $F_{2,3, i}(0)=1$ for $i=1,2,3$, uniquely determine $F_{2,3, i}(a)$ as a double power series in a and $q$.

Proof:

$$
F_{2,3,1}(a)=\frac{1}{1-a q} F_{2,3,3}\left(a q^{2}\right)
$$


is clear, so (26) is immediate. Next,

$$
\begin{aligned}
F_{2,3,2}(a)-F_{2,3,1}(a) & =\sum_{n=0}^{\infty} \frac{a^{n} q^{n^{2}+n}}{\left(a q ; q^{2}\right)_{n+1}(q ; q)_{n}}\left(1-q^{n}\right) \\
& =\sum_{n=1}^{\infty} \frac{a^{n} q^{n^{2}+n}}{\left(a q ; q^{2}\right)_{n+1}(q ; q)_{n-1}} \\
& =\sum_{n=0}^{\infty} \frac{a^{n+1} q^{(n+1)^{2}+(n+1)}}{\left(a q ; q^{2}\right)_{n+2}(q ; q)_{n}} \\
& =\frac{a q^{2}}{1-a q} \sum_{n=0}^{\infty} \frac{a^{n} q^{n^{2}+3 n}}{\left(a q^{3} ; q^{2}\right)_{n+1}(q ; q)_{n}} \\
& =\frac{a q^{2}}{1-a q} F_{2,3,2}\left(a q^{2}\right),
\end{aligned}
$$

so (27) is established. Establishing (28) is a bit trickier, and requires us to define a "catalyst" function

$$
\begin{aligned}
& \phi(a):=\sum_{n=0}^{\infty} \frac{a^{n+1} q^{n^{2}+3 n+1}}{\left(a q ; q^{2}\right)_{n+1}(q ; q)_{n}} . \\
& \frac{a^{2} q^{4}}{1-a q} F_{2,3,1}\left(a q^{2}\right)+\phi(a) \\
= & \frac{a^{2} q^{4}}{1-a q} \sum_{n=0}^{\infty} \frac{a^{n} q^{n^{2}+4 n}}{\left(a q^{3} ; q^{2}\right)_{n+1}(q ; q)_{n}}+\sum_{n=0}^{\infty} \frac{a^{n+1} q^{n^{2}+3 n+1}}{\left(a q ; q^{2}\right)_{n+1}(q ; q)_{n}} \\
= & \sum_{n=0}^{\infty} \frac{a^{n+2} q^{(n+2)^{2}}}{\left(a q ; q^{2}\right)_{n+2}(q ; q)_{n}}+\sum_{n=0}^{\infty} \frac{a^{n+1} q^{n^{2}+3 n+1}}{\left(a q ; q^{2}\right)_{n+1}(q ; q)_{n}} \\
= & \sum_{n=1}^{\infty} \frac{a^{n+1} q^{(n+1)^{2}}}{\left(a q ; q^{2}\right)_{n+1}(q ; q)_{n-1}}+\sum_{n=0}^{\infty} \frac{a^{n+1} q^{n^{2}+3 n+1}}{\left(a q ; q^{2}\right)_{n+1}(q ; q)_{n}} \\
= & \sum_{n=0}^{\infty} \frac{a^{n+1} q^{n^{2}+2 n+1}}{\left(a q ; q^{2}\right)_{n+1}(q ; q)_{n}}\left(1-q^{n}\right)+\sum_{n=0}^{\infty} \frac{a^{n+1} q^{n^{2}+3 n+1}}{\left(a q ; q^{2}\right)_{n+1}(q ; q)_{n}} \\
= & \sum_{n=0}^{\infty} \frac{a^{n+1} q^{n^{2}+2 n+1}}{\left(a q ; q^{2}\right)_{n+1}(q ; q)_{n}}-\sum_{n=0}^{\infty} \frac{a^{n+1} q^{n^{2}+3 n+1}}{\left(a q ; q^{2}\right)_{n+1}(q ; q)_{n}}+\sum_{n=0}^{\infty} \frac{a^{n+1} q^{n^{2}+3 n+1}}{\left(a q ; q^{2}\right)_{n+1}(q ; q)_{n}} \\
= & \sum_{n=0}^{\infty} \frac{a^{n+1} q^{n^{2}+2 n+1}}{\left(a q ; q^{2}\right)_{n+1}(q ; q)_{n}} \\
= & \sum_{n=0}^{\infty} \frac{a^{n} q^{n^{2}}}{\left(a q ; q^{2}\right)_{n}(q ; q)_{n-1}} \\
= & \sum_{n=0}^{\infty} \frac{a^{n} q^{n^{2}}\left(1-a q^{2 n+1}\right)\left(1-q^{n}\right)}{\left(a q ; q^{2}\right)_{n+1}(q ; q)_{n}}
\end{aligned}
$$




$$
\begin{aligned}
& =\sum_{n=0}^{\infty} \frac{a^{n} q^{n^{2}}\left(1-a q^{2 n+1}-q^{n}+a q^{3 n+1}\right)}{\left(a q ; q^{2}\right)_{n+1}(q ; q)_{n}} \\
& =\sum_{n=0}^{\infty} \frac{a^{n} q^{n^{2}}}{\left(a q ; q^{2}\right)_{n}(q ; q)_{n}}\left(1-\frac{q^{n}}{1-a q^{2 n+1}}\right)+\sum_{n=0}^{\infty} \frac{a^{n+1} q^{n^{2}+3 n+1}}{\left(a q ; q^{2}\right)_{n+1}(q ; q)_{n}} \\
& =F_{2,3,3}(a)-F_{2,3,2}(a)+\phi(a),
\end{aligned}
$$

and thus (28) is established.

Thus, combining Lemma 3.8 with Theorem 3.1, we have established the following theorem:

Theorem 3.9 For $i=1,2,3$,

$$
F_{2,3, i}(a)=Q_{2,3, i}(a) .
$$

By setting $a=1$ and employing Proposition 3.3, we obtain three identities of Rogers ( [20] and [21]), which appear as (59), (60), and (61) respectively on Slater's list [26]:

\section{Corollary 3.10}

$$
\begin{aligned}
& \sum_{n=0}^{\infty} \frac{q^{n^{2}+2 n}}{\left(q ; q^{2}\right)_{n+1}(q ; q)_{n}}=\frac{\left(q^{2}, q^{12}, q^{14} ; q^{14}\right)}{(q ; q)_{\infty}} \\
& \sum_{n=0}^{\infty} \frac{q^{n^{2}+n}}{\left(q ; q^{2}\right)_{n+1}(q ; q)_{n}}=\frac{\left(q^{4}, q^{10}, q^{14} ; q^{14}\right)}{(q ; q)_{\infty}} \\
& \sum_{n=0}^{\infty} \frac{q^{n^{2}}}{\left(q ; q^{2}\right)_{n}(q ; q)_{n}}=\frac{\left(q^{6}, q^{8}, q^{14} ; q^{14}\right)}{(q ; q)_{\infty}}
\end{aligned}
$$

\subsubsection{The case $(d, k)=(2,4)$}

Definition 5.

$$
\begin{aligned}
F_{2,4,1}(a) & :=F_{2,4,1}(a, q):=\sum_{n \geqq 0} \sum_{r \geqq 0} \frac{a^{n+r} q^{n^{2}+2 n+2 r^{2}+2 r}}{\left(a q ; q^{2}\right)_{n+1}(q ; q)_{n-2 r}\left(q^{2} ; q^{2}\right)_{r}} \\
F_{2,4,2}(a) & :=F_{2,4,2}(a, q):=\sum_{n \geqq 0} \sum_{r \geqq 0} \frac{a^{n+r} q^{n^{2}+2 n+2 r^{2}+2 r}\left(1+a q^{2 r+2}\right)}{\left(a q ; q^{2}\right)_{n+1}(q ; q)_{n-2 r}\left(q^{2} ; q^{2}\right)_{r}} \\
F_{2,4,3}(a) & :=F_{2,4,3}(a, q):=\sum_{n \geqq 0} \sum_{r \geqq 0} \frac{a^{n+r} q^{n^{2}+2 r^{2}+2 r}}{\left(a q ; q^{2}\right)_{n}(q ; q)_{n-2 r}\left(q^{2} ; q^{2}\right)_{r}} \\
F_{2,4,4}(a) & :=F_{2,4,4}(a, q):=\sum_{n \geqq 0} \sum_{r \geqq 0} \frac{a^{n+r} q^{n^{2}+2 r^{2}}}{\left(a q ; q^{2}\right)_{n}(q ; q)_{n-2 r}\left(q^{2} ; q^{2}\right)_{r}}
\end{aligned}
$$


Lemma 3.11 The $F_{2,4, i}(a, q)$ satisfy the following q-difference equations:

$$
\begin{gathered}
F_{2,4,1}(a)=\frac{1}{1-a q} F_{2,4,4}\left(a q^{2}\right) \\
F_{2,4,2}(a)=F_{2,4,1}(a)+\frac{a q^{2}}{1-a q} F_{2,4,3}\left(a q^{2}\right) \\
F_{2,4,3}(a)=F_{2,4,2}(a)+\frac{a^{2} q^{4}}{1-a q} F_{2,4,2}\left(a q^{2}\right) \\
F_{2,4,4}(a)=F_{2,4,3}(a)+\frac{a^{3} q^{6}}{1-a q} F_{2,4,1}\left(a q^{2}\right),
\end{gathered}
$$

which, together with $F_{2,4, i}(0)=1$ for $i=1,2,3,4$, uniquely determine $F_{2,4, i}(a)$ as a double power series in a and $q$.

If the reader has been following along carefully, the details of the calculations should by now be routine, so I choose to omit the proof of this and subsequent lemmas establishing the $q$-difference equations satisfied by the various $F_{d, k, i}(a)$.

Thus, combining Lemma 3.11 with Theorem 3.1, we have established the following theorem:

Theorem 3.12 For $i=1,2,3,4$,

$$
F_{2,4, i}(a)=Q_{2,4, i}(a)
$$

By setting $a=1$ and employing Proposition 3.3, we obtain four new Rogers-Ramanujan type identities related to the modulus 18 , listed as (A.4)-(A.7) in the appendix.

3.2.4. The case $(d, k)=(3,3)$

Definition 6.

$$
\begin{gathered}
F_{3,3,1}(a):=\sum_{n \geqq 0} \sum_{r \geqq 0} \frac{(-1)^{r} a^{n} q^{n^{2}+3 n+3 r(r-1) / 2}\left(a q^{3} ; q^{3}\right)_{n-r}}{(a q ; q)_{2 n+2}(q ; q)_{n-3 r}\left(q^{3} ; q^{3}\right)_{r}} \\
F_{3,3,2}(a):=\sum_{n \geqq 0} \sum_{r \geqq 0} \frac{(-1)^{r} a^{n-1} q^{n^{2}+3 r(r-3) / 2}\left(a ; q^{3}\right)_{n-r}\left(1+a q^{3 r}-q^{3 r}\right)}{(a ; q)_{2 n}(q ; q)_{n-3 r}\left(q^{3} ; q^{3}\right)_{r}} \\
F_{3,3,3}(a):=\sum_{n \geqq 0} \sum_{r \geqq 0} \frac{(-1)^{r} a^{n} q^{n^{2}+3 r(r-1) / 2}\left(a ; q^{3}\right)_{n-r}}{(a ; q)_{2 n-1}(q ; q)_{n-3 r}\left(q^{3} ; q^{3}\right)_{r}}
\end{gathered}
$$


Lemma 3.13 The $F_{3,3, i}(a, q)$ satisfy the following q-difference equations:

$$
\begin{gathered}
F_{3,3,1}(a)=\frac{1}{(1-a q)\left(1-a q^{2}\right)} F_{3,3,3}\left(a q^{3}\right) \\
F_{3,3,2}(a)=F_{3,3,1}(a)+\frac{a q^{3}}{(1-a q)\left(1-a q^{2}\right)} F_{3,3,2}\left(a q^{3}\right) \\
F_{3,3,3}(a)=F_{3,3,2}(a)+\frac{a^{2} q^{6}}{(1-a q)\left(1-a q^{2}\right)} F_{3,3,1}\left(a q^{3}\right),
\end{gathered}
$$

which, together with $F_{3,3, i}(0)=1$ for $i=1,2,3$, uniquely determine $F_{3,3, i}(a)$ as a double power series in $a$ and $q$.

Thus, combining Lemma 3.13 with Theorem 3.1, we have established the following theorem:

Theorem 3.14 For $i=1,2,3$,

$$
F_{3,3, i}(a)=Q_{3,3, i}(a)
$$

As an immediate corollary, by letting $a \rightarrow 1$, we obtain three new Rogers-Ramanujan type identities related to the modulus 21 , listed as (A.8)-(A.10) in the appendix.

3.2.5. The case $(d, k)=(3,4)$

Definition 7.

$$
\begin{aligned}
F_{3,4,1}(a) & :=F_{3,4,1}(a, q):=\sum_{n=0}^{\infty} \frac{a^{n} q^{n(n+3)}\left(a q^{3} ; q^{3}\right)_{n}}{(a q ; q)_{2 n+2}(q ; q)_{n}} \\
F_{3,4,2}(a) & :=F_{3,4,2}(a, q):=\sum_{n=0}^{\infty} \frac{a^{n} q^{n(n+2)}\left(a q^{3} ; q^{3}\right)_{n}}{(a q ; q)_{2 n+2}(q ; q)_{n}} \\
F_{3,4,3}(a) & :=F_{3,4,3}(a, q):=\sum_{n=0}^{\infty} \frac{a^{n} q^{n(n+1)}\left(a q^{3} ; q^{3}\right)_{n}}{(a q ; q)_{2 n+1}(q ; q)_{n}} \\
F_{3,4,4}(a) & :=F_{3,4,4}(a, q):=\sum_{n=0}^{\infty} \frac{a^{n} q^{n^{2}}\left(a ; q^{3}\right)_{n}}{(a ; q)_{2 n}(q ; q)_{n}}
\end{aligned}
$$


Lemma 3.15 The $F_{3,4, i}(a, q)$ satisfy the following q-difference equations:

$$
\begin{gathered}
F_{3,4,1}(a)=\frac{1}{(1-a q)\left(1-a q^{2}\right)} F_{3,4,4}\left(a q^{3}\right) \\
F_{3,4,2}(a)=F_{3,4,1}(a)+\frac{a q^{3}}{(1-a q)\left(1-a q^{2}\right)} F_{3,4,3}\left(a q^{3}\right) \\
F_{3,4,3}(a)=F_{3,4,2}(a)+\frac{a^{2} q^{6}}{(1-a q)\left(1-a q^{2}\right)} F_{3,4,2}\left(a q^{3}\right) \\
F_{3,4,4}(a)=F_{3,4,3}(a)+\frac{a^{3} q^{9}}{(1-a q)\left(1-a q^{2}\right)} F_{3,4,1}\left(a q^{3}\right),
\end{gathered}
$$

which, together with $F_{3,4, i}(0)=1$ for $i=1,2,3,4$, uniquely determine $F_{3,4, i}(a)$ as a double power series in $a$ and $q$.

Theorem 3.16 For $i=1,2,3,4$,

$$
F_{3,4, i}(a)=Q_{3,4, i}(a)
$$

Upon letting $a \rightarrow 1$ and employing Proposition 3.3, we obtain the Bailey-Dyson mod 27 identities [8, p. 434, equations (B1)-(B4)], which appear as (90)-(93) on Slater's list [26].

\section{Corollary 3.17}

$$
\begin{gathered}
\sum_{n=0}^{\infty} \frac{q^{n(n+3)}\left(q^{3} ; q^{3}\right)_{n}}{(q ; q)_{2 n+2}(q ; q)_{n}}=\frac{\left(q^{3}, q^{24}, q^{27} ; q^{27}\right)_{\infty}}{(q ; q)_{\infty}} \\
\sum_{n=0}^{\infty} \frac{q^{n(n+2)}\left(q^{3} ; q^{3}\right)_{n}}{(q ; q)_{2 n+2}(q ; q)_{n}}=\frac{\left(q^{6}, q^{18}, q^{27} ; q^{27}\right)_{\infty}}{(q ; q)_{\infty}} \\
\sum_{n=0}^{\infty} \frac{q^{n(n+1)}\left(q^{3} ; q^{3}\right)_{n}}{(q ; q)_{2 n+1}(q ; q)_{n}}=\frac{\left(q^{9} ; q^{9}\right)_{\infty}}{(q ; q)_{\infty}} \\
1+\sum_{n=1}^{\infty} \frac{q^{n^{2}}\left(q^{3} ; q^{3}\right)_{n-1}}{(q ; q)_{2 n-1}(q ; q)_{n}}=\frac{\left(q^{12}, q^{15}, q^{27} ; q^{27}\right)_{\infty}}{(q ; q)_{\infty}}
\end{gathered}
$$

3.2.6. The case $(d, k)=(3,5)$ 
Definition 8.

$$
\begin{gathered}
F_{3,5,1}(a):=\sum_{n \geqq 0} \sum_{r \geqq 0} \frac{a^{n+r} q^{n^{2}+3 r^{2}+3 n+3 r}\left(a q^{3} ; q^{3}\right)_{n-r}}{(a q ; q)_{2 n+2}(q ; q)_{n-3 r}\left(q^{3} ; q^{3}\right)_{r}} \\
F_{3,5,2}(a):=\sum_{n \geqq 0} \sum_{r \geqq 0} \frac{a^{n+r} q^{n^{2}+3 r^{2}+3 n+3 r}\left(a q^{3} ; q^{3}\right)_{n-r}\left(1+a q^{3 r+3}\right)}{(a q ; q)_{2 n+2}(q ; q)_{n-3 r}\left(q^{3} ; q^{3}\right)_{r}} \\
F_{3,5,3}(a):=\sum_{n \geqq 0} \sum_{r \geqq 0} \frac{a^{n+r-1} q^{n^{2}+3 r^{2}-3}\left(a ; q^{3}\right)_{n-r}\left(q^{3 r}+a q^{6 r+3}-1\right)}{(a ; q)_{2 n}(q ; q)_{n-3 r}\left(q^{3} ; q^{3}\right)_{r}} \\
F_{3,5,4}(a):=\sum_{n \geqq 0} \sum_{r \geqq 0} \frac{a^{n+r} q^{n^{2}+3 r^{2}+3 r}\left(a ; q^{3}\right)_{n-r}}{(a ; q)_{2 n}(q ; q)_{n-3 r}\left(q^{3} ; q^{3}\right)_{r}} \\
F_{3,5,5}(a):=\sum_{n \geqq 0} \sum_{r \geqq 0} \frac{a^{n+r} q^{n^{2}+3 r^{2}}\left(a ; q^{3}\right)_{n-r}}{(a ; q)_{2 n}(q ; q)_{n-3 r}\left(q^{3} ; q^{3}\right)_{r}}
\end{gathered}
$$

Lemma 3.18 The $F_{3,5, i}(a, q)$ satisfy the following q-difference equations:

$$
\begin{gathered}
F_{3,5,1}(a)=\frac{1}{(1-a q)\left(1-a q^{2}\right)} F_{3,5,5}\left(a q^{3}\right) \\
F_{3,5,2}(a)=F_{3,5,1}(a)+\frac{a q^{3}}{(1-a q)\left(1-a q^{2}\right)} F_{3,5,4}\left(a q^{3}\right) \\
F_{3,5,3}(a)=F_{3,5,2}(a)+\frac{a^{2} q^{6}}{(1-a q)\left(1-a q^{2}\right)} F_{3,5,3}\left(a q^{3}\right) \\
F_{3,5,4}(a)=F_{3,5,3}(a)+\frac{a^{3} q^{9}}{(1-a q)\left(1-a q^{2}\right)} F_{3,5,2}\left(a q^{3}\right) \\
F_{3,5,5}(a)=F_{3,5,4}(a)+\frac{a^{4} q^{12}}{(1-a q)\left(1-a q^{2}\right)} F_{3,5,1}\left(a q^{3}\right)
\end{gathered}
$$

which, together with $F_{3,5, i}(0)=1$ for $i=1,2,3,4,5$, uniquely determine $F_{3,5, i}(a)$ as a double power series in a and $q$.

Theorem 3.19 For $i=1,2,3,4,5$,

$$
F_{3,5, i}(a)=Q_{3,5, i}(a)
$$

Upon letting $a \rightarrow 1$ and employing Proposition 3.3, we obtain five mod 33 identities listed in the appendix as (A.16) through (A.20).

\section{Partition Theorems}

In 1961, Basil Gordon [15] published an infinite family of partition identities which generalized the combinatorial version of the Rogers-Ramanujan identities: 
Gordon's Partition Theorem Let $B_{1, k, i}(n)$ denote the number of partitions of $n$ wherein 1 appears as a part at most $i-1$ times, and the total number of appearances of any two consecutive integers $j$ and $j+1$ is at most $k-1$. Let $A_{1, k, i}(n)$ denote the number of partitions of $n$ into parts not congruent to 0 or $\pm i(\bmod 2 k+1)$. Then $A_{1, k, i}(n)=B_{1, k, i}(n)$ for all $n$ and $1 \leqq i \leqq k$.

Later, George Andrews [3] found an analytic counterpart to Gordon's partition theorem:

Andrews' Analytic Counterpart to Gordon's Theorem For $1 \leqq i \leqq k$ and $k \geqq 2$,

$$
\sum_{n_{1}, n_{2}, \ldots, n_{k-1} \geqq 0} \frac{q^{\sum_{j=1}^{k-1} N_{j}^{2}+\sum_{j=i}^{k-1} N_{j}}}{(q ; q)_{n_{1}}(q ; q)_{n_{2}} \ldots(q ; q)_{n_{k-1}}}=\prod_{\substack{n=0, \pm i \\(\bmod 2 k+1)}}^{\infty} \frac{1}{1-q^{n}}
$$

where $N_{j}=\sum_{h=j}^{k-1} n_{h}$.

Motivated by the analytic results earlier in this paper, we consider Theorem 1.1, restated here for convenience.

Theorem 4.1 Let $A_{d, k, i}(n)$ denote the number of partitions of $n$ into parts $\not \equiv 0, \pm d i$ $(\bmod 2 d k+d)$. Let $B_{d, k, i}(n)$ denote the number of partitions of $n$ wherein

- $d$ appears as a part at most $i-1$ times,

- the total number of appearances of $d j$ and $d j+d$ (i.e. any two consecutive multiples of d) together is at most $k-1$, and

- nonmultiples of d may appear as parts without restriction.

Then for $1 \leqq i \leqq k, A_{d, k, i}(n)=B_{d, k, i}(n)$.

Remark. Clearly, the case $d=1$ is Gordon's partition theorem.

Proof:

$$
\begin{aligned}
\sum_{n=0}^{\infty} B_{d, k, i}(n) q^{n} & =\prod_{\substack{j=1 \\
d \nmid j}}^{\infty} \frac{1}{1-q^{j}} \sum_{n=0}^{\infty} B_{1, k, i}(d n) q^{n d} \\
& =\prod_{\substack{j=1 \\
d \nmid j}}^{\infty} \frac{1}{1-q^{j}} \times \prod_{\substack{j=1 \\
(\bmod (2 k+1))}}^{\infty} \frac{1}{1-q^{d j}} \\
& =\prod_{\substack{j \neq 0, \pm d i \\
(\bmod (2 k+1) d)}}^{\infty} \frac{1}{1-q^{j}}
\end{aligned}
$$

Definition 9. Let $b_{d, k, i}(m, n)$ denote the number of partitions of $n$ of the kind enumerated by $B_{d, k, i}(n)$ with the further restriction that the partition contains exactly $m$ parts. 
Definition 10.

$$
\mathcal{B}_{d, k, i}(a):=\mathcal{B}_{d, k, i}(a, q):=\sum_{m, n \geqq 0} b_{d, k, i}(m, n) a^{m} q^{n} .
$$

Theorem 4.2 The $\mathcal{B}_{d, k, i}(a)$ satisfy the following system of q-difference equations:

$$
\begin{aligned}
\mathcal{B}_{d, k, 1}(a) & =\frac{1}{(a q ; q)_{d-1}} \mathcal{B}_{d, k, k}\left(a q^{d}\right) \\
\mathcal{B}_{d, k, i}(a) & =\mathcal{B}_{k, d, i-1}(a)+\frac{a^{i-1} q^{(i-1) d}}{(a q ; q)_{d-1}} \mathcal{B}_{d, k, k-i+1}\left(a q^{d}\right),
\end{aligned}
$$

for $2 \leqq i \leqq k$.

Proof: To obtain partitions of the type enumerated by $b_{d, k, 1}(m, n)$ from those enumerated by $b_{d, k, k}(m, n)$, one simply needs to increase each part in the latter class by $d$ and adjoin as many 1's, 2's, $\ldots$, and ( $d-1)$ 's as desired. Thus, (37) holds.

Now let us segregate the partitions generated by $b_{d, k, i}(m, n)$ into two classes: those where $d$ appears as a part at most $i-2$ times and those where $d$ appears exactly $i-1$ times. Those in the former class are the entire set of partitions enumerated by $b_{d, k, i-1}(m, n)$. Those in the latter class may be obtained by starting with the set of partitions enumerated by $b_{d, k, k-i+1}$, increasing each part by $d$, and affixing exactly $i-1$ copies of the part $d$, and as many 1 's, 2 's, $\ldots$, and $(d-1)$ 's as desired. Thus, (38) holds.

Since $\mathcal{B}_{d, k, i}(0)$ for $1 \leqq i \leqq k$, by uniqueness of power series, we immediately obtain

\section{Corollary 4.3}

$$
\mathcal{B}_{d, k, i}(a)=Q_{d, k, i}(a)
$$

for all $d$, all $k$, and $1 \leqq i \leqq k$, and

$$
\mathcal{B}_{d, k, i}(a)=Q_{d, k, i}(a)=F_{d, k, i}(a)
$$

for $(d, k)=(2,2),(2,3),(2,4),(3,3),(3,4),(3,5)$, where $1 \leqq i \leqq k$.

As a corollary of Corollary 4.3, by setting $a=1$, and in light of (3.3), we obtain combinatorial interpretations of a variety of identities in Slater's list, as well as some of the new identities presented in the appendix. For example, the statement

$$
\mathcal{B}_{2,3, i}(1)=Q_{2,3, i}(1)
$$

provides the partition theoretic interpretation of the Rogers mod 14 identities (29)-(31), which was stated in the introduction as Corollary 1.2. Of course, similar partition theoretic statements can be made for all other values of $d$ and $k$, and can be seen as the combinatorial counterparts to the $a=1$ case of the various $F_{d, k, i}(a)=Q_{d, k, i}(a)$ identities presented in $\S 3$. 


\section{Conclusion}

This paper was motivated by taking a careful second look at the methods employed by Bailey ([8],[9]) and seeing if they could be pushed a bit farther. Notice that only classical techniques (Bailey's Lemma, transformations basic hypergeometric series, and $q$-difference equations) were used. One of the goals of this paper is to illustrate that even after all these years, many stones remain unturned along the Rogers-Ramanujan path, even when only classical methods are used.

Presumably the methods of this paper could be used to obtain additional identities for other values of $d$ and $k$. For instance if $d+k=7$, the expression for $\beta_{m}(a, q)$ will involve a ${ }_{10} W_{9}$, which could be transformed into a double sum expression (see [4]), ultimately yielding a triple sum-product identity.

Also, considering the sets of identities produced when instances of the parametrized Bailey pair in Theorem 2 are inserted into (10), it seems reasonable that the associated identities could be related to a " $d$-extended" version of Andrews' combinatorial generalization of the Göllnitz-Gordon partition theorem [1], analogous to Theorem 1.1. Likewise, it is plausible that the identities arising in connection with (11) could be explained combinatorially using the overpartitions studied recently by Corteel and Lovejoy ([13], [16]).

Furthermore, the technique of obtaining parametrized Bailey pairs could presumably be applied to other $\alpha$ 's such as the one from which the Rogers-Selberg identities [9, p. 5, (ii)] or Bailey's mod 9 identities [9, p. 5, (iii)] are derived, yielding other families of results.

Additionally, finite analogs of Rogers-Ramanujan type identities have, in recent years, been of great interest in physics (e.g. [7], [10], [11], [12], [22], [27], [28], [29]) and symbolic computation (e.g. [18], [19], [30], [31]). In a recent paper [24], I presented finite analogs for all of the identities in Slater's list. The conjecture and proof of these polynomial identities relied heavily on the use of computer algebra [25]. It is therefore natural to ask whether the techniques successfully employed for finitizing the single sum-product identities of Slater's list can be extended to the double sum identities presented here, and more generally to arbitrary multisum-product identities.

\section{Acknowledgement}

I thank the referee for a thorough, careful reading of the manuscript, and for the many helpful comments.

\section{Appendix}

\section{A List of Double Sum Identities of the Rogers-Ramanujan Type}

The following are immediate consequences of the more general results presented earlier in the paper.

For $(d, k)=(2,1)$, insert (13) into (11):

$$
\sum_{n \geqq 0} \sum_{r \geqq 0} \frac{(-1)^{r} q^{3 n(n-1) / 2+r^{2}-2 n r}}{\left(q ; q^{2}\right)_{n}\left(q^{2} ; q^{2}\right)_{r}(q ; q)_{n-2 r}}=\frac{\left(q^{2}, q^{4}, q^{6} ; q^{6}\right)_{\infty}}{(q ; q)_{\infty}}=(-q ; q)_{\infty}
$$


For $(d, k)=(3,3)$, insert (14) into (11):

$$
\begin{gathered}
1+\sum_{n \geqq 1} \sum_{r \geqq 0} \frac{(-1)^{r} q^{n(n+1) / 2+3 r(r-1) / 2}(-1 ; q)_{n}\left(q^{3} ; q^{3}\right)_{n-r-1}}{(q ; q)_{2 n-1}\left(q^{3} ; q^{3}\right)_{r}(q ; q)_{n-3 r}} \\
=\frac{\left(q^{6}, q^{6}, q^{12} ; q^{12}\right)_{\infty}(-q ; q)_{\infty}}{(q ; q)_{\infty}}
\end{gathered}
$$

For $(d, k)=(2,4)$, insert (12) into (11):

$$
\sum_{n \geqq 0} \sum_{r \geqq 0} \frac{q^{n(n+1) / 2+2 r^{2}}(-1 ; q)_{n}}{\left(q ; q^{2}\right)_{n}\left(q^{2} ; q^{2}\right)_{r}(q ; q)_{n-2 r}}=\frac{\left(q^{7}, q^{7}, q^{14} ; q^{14}\right)_{\infty}(-q ; q)_{\infty}}{(q ; q)_{\infty}}
$$

...into (9):

$$
\begin{aligned}
& \sum_{n \geqq 0} \sum_{r \geqq 0} \frac{q^{n^{2}+2 n+2 r^{2}+2 r}}{\left(q ; q^{2}\right)_{n+1}\left(q^{2} ; q^{2}\right)_{r}(q ; q)_{n-2 r}}=\frac{\left(q^{2}, q^{16}, q^{18} ; q^{18}\right)_{\infty}}{(q ; q)_{\infty}} \\
& \sum_{n \geqq 0} \sum_{r \geqq 0} \frac{q^{n^{2}+2 n+2 r^{2}+2 r}\left(1+q^{2 r+2}\right)}{\left(q ; q^{2}\right)_{n+1}\left(q^{2} ; q^{2}\right)_{r}(q ; q)_{n-2 r}}=\frac{\left(q^{4}, q^{14}, q^{18} ; q^{18}\right)_{\infty}}{(q ; q)_{\infty}} \\
& \sum_{n \geqq 0} \sum_{r \geqq 0} \frac{q^{n^{2}+2 r^{2}+2 r}}{\left(q ; q^{2}\right)_{n}\left(q^{2} ; q^{2}\right)_{r}(q ; q)_{n-2 r}}=\frac{\left(q^{6}, q^{12}, q^{18} ; q^{18}\right)_{\infty}}{(q ; q)_{\infty}} \\
& \sum_{n \geqq 0} \sum_{r \geqq 0} \frac{q^{n^{2}+2 r^{2}}}{\left(q ; q^{2}\right)_{n}\left(q^{2} ; q^{2}\right)_{r}(q ; q)_{n-2 r}}=\frac{\left(q^{8}, q^{10}, q^{18} ; q^{18}\right)_{\infty}}{(q ; q)_{\infty}}
\end{aligned}
$$

For $(d, k)=(3,3)$, insert (14) into (9):

$$
\begin{array}{r}
\sum_{n \geqq 0} \sum_{r \geqq 0} \frac{(-1)^{r} q^{n^{2}+3 n+3 r(r-1) / 2}\left(q^{3} ; q^{3}\right)_{n-r}}{(q ; q)_{2 n+2}\left(q^{3} ; q^{3}\right)_{r}(q ; q)_{n-3 r}}=\frac{\left(q^{3}, q^{18}, q^{21} ; q^{21}\right)_{\infty}}{(q ; q)_{\infty}} \\
1+\sum_{n \geqq 1} \sum_{r \geqq 0} \frac{(-1)^{r} q^{n^{2}+3 r(r-3) / 2}\left(q^{3} ; q^{3}\right)_{n-r-1}}{(q ; q)_{2 n-1}\left(q^{3} ; q^{3}\right)_{r}(q ; q)_{n-3 r}}=\frac{\left(q^{6}, q^{15}, q^{21} ; q^{21}\right)_{\infty}}{(q ; q)_{\infty}} \\
1+\sum_{n \geqq 1} \sum_{r \geqq 0} \frac{(-1)^{r} q^{n^{2}+3 r(r-1) / 2}\left(q^{3} ; q^{3}\right)_{n-r-1}}{(q ; q)_{2 n-1}\left(q^{3} ; q^{3}\right)_{r}(q ; q)_{n-3 r}}=\frac{\left(q^{9}, q^{12}, q^{21} ; q^{21}\right)_{\infty}}{(q ; q)_{\infty}}
\end{array}
$$

...into (10):

$1+\sum_{n \geqq 1} \sum_{r \geqq 0} \frac{(-1)^{r} q^{n^{2}+3 r(r-3)}\left(-q ; q^{2}\right)_{n}\left(q^{6} ; q^{6}\right)_{n-r-1}}{\left(q^{2} ; q^{2}\right)_{2 n-1}\left(q^{6} ; q^{6}\right)_{r}\left(q^{2} ; q^{2}\right)_{n-3 r}}=\frac{\left(q^{3}, q^{21}, q^{24} ; q^{24}\right)_{\infty}\left(-q ; q^{2}\right)_{\infty}}{\left(q^{2} ; q^{2}\right)_{\infty}}$ 
$1+\sum_{n \geqq 1} \sum_{r \geqq 0} \frac{(-1)^{r} q^{n^{2}+3 r(r-1)}\left(-q ; q^{2}\right)_{n}\left(q^{6} ; q^{6}\right)_{n-r-1}}{\left(q^{2} ; q^{2}\right)_{2 n-1}\left(q^{6} ; q^{6}\right)_{r}\left(q^{2} ; q^{2}\right)_{n-3 r}}=\frac{\left(q^{9}, q^{15}, q^{24} ; q^{24}\right)_{\infty}\left(-q ; q^{2}\right)_{\infty}}{\left(q^{2} ; q^{2}\right)_{\infty}}$

For $(d, k)=(3,5)$, insert (15) into (11):

$$
1+\sum_{n \geqq 1} \sum_{r \geqq 0} \frac{q^{n(n+1) / 2+3 r^{2}}(-1 ; q)_{n}\left(q^{3} ; q^{3}\right)_{n-r-1}}{(q ; q)_{2 n-1}\left(q^{3} ; q^{3}\right)_{r}(q ; q)_{n-3 r}}=\frac{\left(q^{12}, q^{12}, q^{24} ; q^{24}\right)_{\infty}(-q ; q)_{\infty}}{(q ; q)_{\infty}}
$$

For $(d, k)=(2,4)$, insert (12) into (10):

$$
\begin{aligned}
& \sum_{n \geqq 0} \sum_{r \geqq 0} \frac{q^{n^{2}+4 r^{2}+4 r}}{\left(q ; q^{2}\right)_{n}\left(q^{4} ; q^{4}\right)_{r}\left(q^{2} ; q^{2}\right)_{n-2 r}}=\frac{\left(q^{8}, q^{20}, q^{28} ; q^{28}\right)_{\infty}\left(-q ; q^{2}\right)_{\infty}}{\left(q^{2} ; q^{2}\right)_{\infty}} \\
& \sum_{n \geqq 0} \sum_{r \geqq 0} \frac{q^{n^{2}+4 r^{2}}}{\left(q ; q^{2}\right)_{n}\left(q^{4} ; q^{4}\right)_{r}\left(q^{2} ; q^{2}\right)_{n-2 r}}=\frac{\left(q^{12}, q^{16}, q^{28} ; q^{28}\right)_{\infty}\left(-q ; q^{2}\right)_{\infty}}{\left(q^{2} ; q^{2}\right)_{\infty}}
\end{aligned}
$$

For $(d, k)=(3,5)$, insert (15) into (9):

$$
\begin{gathered}
\sum_{n \geqq 0} \sum_{r \geqq 0} \frac{q^{n^{2}+3 n+3 r^{2}+3 r}\left(q^{3} ; q^{3}\right)_{n-r}}{(q ; q)_{2 n+2}\left(q^{3} ; q^{3}\right)_{r}(q ; q)_{n-3 r}}=\frac{\left(q^{3}, q^{30}, q^{33} ; q^{33}\right)_{\infty}}{(q ; q)_{\infty}} \\
\sum_{n \geqq 0} \sum_{r \geqq 0} \frac{q^{n^{2}+3 n+3 r^{2}+3 r}\left(q^{3} ; q^{3}\right)_{n-r}\left(1+q^{3 r+3}\right)}{(q ; q)_{2 n+2}\left(q^{3} ; q^{3}\right)_{r}(q ; q)_{n-3 r}}=\frac{\left(q^{6}, q^{27}, q^{33} ; q^{33}\right)_{\infty}}{(q ; q)_{\infty}} \\
1+\sum_{n \geqq 1} \sum_{r \geqq 0} \frac{q^{n^{2}+3 r^{2}-3}\left(q^{3} ; q^{3}\right)_{n-r-1}\left(q^{3 r}+q^{6 r+3}-1\right)}{(q ; q)_{2 n-1}\left(q^{3} ; q^{3}\right)_{r}(q ; q)_{n-3 r}}=\frac{\left(q^{9}, q^{24}, q^{33} ; q^{33}\right)_{\infty}}{(q ; q)_{\infty}} \\
1+\sum_{n \geqq 1} \sum_{r \geqq 0} \frac{q^{n^{2}+3 r^{2}+3 r}\left(q^{3} ; q^{3}\right)_{n-r-1}}{(q ; q)_{2 n-1}\left(q^{3} ; q^{3}\right)_{r}(q ; q)_{n-3 r}}=\frac{\left(q^{12}, q^{21}, q^{33} ; q^{33}\right)_{\infty}}{(q ; q)_{\infty}} \\
1+\sum_{n \geqq 1} \sum_{r \geqq 0} \frac{q^{n^{2}+3 r^{2}}\left(q^{3} ; q^{3}\right)_{n-r-1}}{(q ; q)_{2 n-1}\left(q^{3} ; q^{3}\right)_{r}(q ; q)_{n-3 r}}=\frac{\left(q^{15}, q^{18}, q^{33} ; q^{33}\right)_{\infty}}{(q ; q)_{\infty}}
\end{gathered}
$$


For $(d, k)=(4,6)$, insert (16) and (17) into (11):

$$
\begin{gathered}
1+\sum_{m \geqq 1} \sum_{r \geqq 0} \frac{(-1)^{m+r} q^{m^{2}+m+r^{2}+r-2 m r}\left(q^{4} ; q^{4}\right)_{m+r-1}(-1 ; q)_{2 m}}{(q ; q)_{4 m-1}(q ; q)_{2 r}\left(q^{2} ; q^{2}\right)_{m-r}} \\
+\sum_{m \geqq 0} \sum_{r \geqq 0} \frac{(-1)^{m+r} q^{m^{2}+m+r^{2}+r-2 m r+1}\left(q^{4} ; q^{4}\right)_{m+r}(-1 ; q)_{2 m+1}}{(q ; q)_{4 m+1}(q ; q)_{2 r+1}\left(q^{2} ; q^{2}\right)_{m-r}} \\
=\frac{\left(q^{18}, q^{18}, q^{36} ; q^{36}\right)_{\infty}(-q ; q)_{\infty}}{(q ; q)_{\infty}}
\end{gathered}
$$

For $(d, k)=(3,5)$, insert (15) into (10):

$$
\begin{gathered}
1+\sum_{n \geqq 1} \sum_{r \geqq 0} \frac{q^{n^{2}+6 r^{2}-6}\left(-q ; q^{2}\right)_{n}\left(q^{6} ; q^{6}\right)_{n-r-1}\left(q^{6 r}+q^{12 r+6}-1\right)}{\left(q^{2} ; q^{2}\right)_{2 n-1}\left(q^{6} ; q^{6}\right)_{r}\left(q^{2} ; q^{2}\right)_{n-3 r}} \\
=\frac{\left(q^{9}, q^{39}, q^{48} ; q^{48}\right)_{\infty}\left(-q ; q^{2}\right)_{\infty}}{\left(q^{2} ; q^{2}\right)_{\infty}} \\
1+\sum_{n \geqq 1} \sum_{r \geqq 0} \frac{q^{n^{2}+6 r^{2}+6 r}\left(-q ; q^{2}\right)_{n}\left(q^{6} ; q^{6}\right)_{n-r-1}}{\left(q^{2} ; q^{2}\right)_{2 n-1}\left(q^{6} ; q^{6}\right)_{r}\left(q^{2} ; q^{2}\right)_{n-3 r}}=\frac{\left(q^{15}, q^{33}, q^{48} ; q^{48}\right)_{\infty}\left(-q ; q^{2}\right)_{\infty}}{\left(q^{2} ; q^{2}\right)_{\infty}} \\
1+\sum_{n \geqq 1} \sum_{r \geqq 0} \frac{q^{n^{2}+6 r^{2}}\left(-q ; q^{2}\right)_{n}\left(q^{6} ; q^{6}\right)_{n-r-1}}{\left(q^{2} ; q^{2}\right)_{2 n-1}\left(q^{6} ; q^{6}\right)_{r}\left(q^{2} ; q^{2}\right)_{n-3 r}}=\frac{\left(q^{21}, q^{27}, q^{48} ; q^{48}\right)_{\infty}\left(-q ; q^{2}\right)_{\infty}}{\left(q^{2} ; q^{2}\right)_{\infty}}
\end{gathered}
$$

For $(d, k)=(4,6)$, insert (16) and (17) into (9):

$$
\begin{gathered}
1+\sum_{m \geqq 1} \sum_{r \geqq 0} \frac{(-1)^{m+r} q^{3 m^{2}+r^{2}+r-2 m r}\left(q^{4} ; q^{4}\right)_{m+r-1}}{(q ; q)_{4 m-1}(q ; q)_{2 r}\left(q^{2} ; q^{2}\right)_{m-r}} \\
+\sum_{m \geqq 0} \sum_{r \geqq 0} \frac{(-1)^{m+r} q^{3 m^{2}+2 m+r^{2}+r-2 m r+1}\left(q^{4} ; q^{4}\right)_{m+r}}{(q ; q)_{4 m+1}(q ; q)_{2 r+1}\left(q^{2} ; q^{2}\right)_{m-r}} \\
=\frac{\left(q^{24}, q^{28}, q^{52} ; q^{52}\right)_{\infty}}{(q ; q)_{\infty}}
\end{gathered}
$$

...into (10):

$$
\begin{aligned}
& 1+\sum_{m \geqq 1} \sum_{r \geqq 0} \frac{(-1)^{m+r} q^{2 m^{2}+2 r^{2}+2 r-4 m r}\left(q^{8} ; q^{8}\right)_{m+r-1}\left(-q ; q^{2}\right)_{2 m}}{\left(q^{2} ; q^{2}\right)_{4 m-1}\left(q^{2} ; q^{2}\right)_{2 r}\left(q^{4} ; q^{4}\right)_{m-r}} \\
& +\sum_{m \geqq 0} \sum_{r \geqq 0} \frac{(-1)^{m+r} q^{2 m^{2}+2 r^{2}+2 r-4 m r+1}\left(q^{8} ; q^{8}\right)_{m+r}\left(-q ; q^{2}\right)_{2 m+1}}{\left(q^{2} ; q^{2}\right)_{4 m+1}\left(q^{2} ; q^{2}\right)_{2 r+1}\left(q^{4} ; q^{4}\right)_{m-r}}
\end{aligned}
$$




$$
=\frac{\left(q^{32}, q^{40}, q^{72} ; q^{72}\right)_{\infty}\left(-q ; q^{2}\right)_{\infty}}{\left(q^{2} ; q^{2}\right)_{\infty}}
$$

\section{References}

1. G. E. Andrews, "A generalization of the Göllnitz-Gordon partition theorems," Proc. Amer. Math. Soc. 18 (1967), no. 5, 945-952.

2. G. E. Andrews, The Theory of Partitions, Addison-Wesley, 1976; reissued Cambridge Univ. Press, 1998.

3. G. E. Andrews, "An analytic generalization of the Rogers-Ramanujan identities for odd moduli," Proc. Nat. Acad. Sci. USA, 71 (1974), 4082-4085.

4. G. E. Andrews, "Problems and Prospects for basic hypergeometric functions," in Theory and Application of Special Functions, (R. Askey ed.), Academic Press, New York, 1975, 191-214.

5. G. E. Andrews, "Multiple series Rogers-Ramanujan type identities," Pacific J. Math., 114 (1984), 267-283.

6. G. E. Andrews, q-series: their development and application in analysis, number theory, combinatorics, physics, and computer algebra, CBMS Regional Conferences Series in Mathematics, no. 66, American Mathematical Society, Providence, RI, 1986.

7. G. E. Andrews, R. J. Baxter, and P. J. Forrester, "Eight vertex SOS model and generalized Rogers-Ramanujan type identities," J. Statist. Phys., 35 (1984), 193-266.

8. W. N. Bailey, "Some identities in combinatory analysis," Proc. London Math. Soc. (2), 49 (1947), 421-435.

9. W. N. Bailey, "Identities of the Rogers-Ramanujan type," Proc. London Math. Soc. (2), 50 (1949), 1-10.

10. A. Berkovich and B. M. McCoy, "Continued fractions and fermionic representations for characters of $M\left(p, p^{\prime}\right)$ minimal models," Lett. Math. Phys., 37 (1996), 49-66.

11. A. Berkovich, B. M. McCoy, and W. P. Orrick, Polynomial identities, indices, and duality for the $N=1$ superconformal model $S M(2,4 \nu)$, J. Statist. Phys., 83 (1996) 795-837.

12. A. Berkovich, B. M. McCoy, and A. Schilling, Rogers-Schur-Ramanujan type identities for the $M\left(p, p^{\prime}\right)$ minimal models of conformal field theory, Comm. Math. Phys., 191 (1998), 211-223.

13. S. Corteel and J. Lovejoy, "Overpartitions," Trans. Amer. Math Soc., 356 (2004), 1623-1635.

14. G. Gasper and M. Rahman, Basic Hypergeometric Series, Cambridge Univ. Press, 1990.

15. B. Gordon, "A combinatorial generalization of the Rogers-Ramanujan identities," Amer. J. Math., 83 (1961), 393-399.

16. J. Lovejoy, “Gordon's theorem for overpartitions," J. Comb. Theory Ser. A, 103 (2003), 393-401.

17. P. A. MacMahon, Combinatory Analysis, vol. 2, Cambridge Univ. Press, London, 1918.

18. P. Paule, "Short and easy computer proofs of the Rogers-Ramanujan identities and of identities of similar type," Electron. J. Combin., 1 (1994), \# R10, 1-9.

19. M. Petkovšek, H. S. Wilf, and D. Zeilberger. A=B. A. K. Peters, Wellesley, MA, 1996.

20. L. J. Rogers, "Second memoir on the expansion of certain infinite products," Proc. London Math Soc. (1), 25 (1894), 318-343.

21. L. J. Rogers, "On two theorems of combinatory analysis and some allied identities," Proc. London Math. Soc. (2), 16 (1917), 315-336.

22. A. Schilling and S. O. Warnaar, "Supernomial coefficients, polynomial identities, and $q$-series," Ramanujan J., 2 (1998), 459-494.

23. I. Schur, "Ein Beitrag zur additiven Zahlentheorie und zur Theorie der Kettenbrüche," Sitzungsberichte der Berliner Akademie (1917), 302-321.

24. A. V. Sills, "Finite Rogers Ramanujan Type Identities," Electronic J. Combin., 10(1) (2003), \# R13, pp. $1-122$.

25. A. V. Sills, "RRtools-a Maple package for the discovery and proof of Rogers-Ramanujan type identities," J. Symbolic Comput., 37 (2004), 415-448.

26. L. J. Slater, "Further identities of the Rogers-Ramanujan type," Proc. London Math Soc. (2), 54 (1952), $147-167$.

27. S. O. Warnaar, "The generalized Borwein conjecture II: refined $q$-trinomial coefficients," Discrete Math., 272 (2003), 215-258.

28. S. O. Warnaar, “ $q$-trinomial identities," J. Math. Phys., 40(4) (1999), 2514-2530.

29. S. O. Warnaar, "Refined $q$-trinomial coefficients and character identities," J. Stat. Phys. 102 (2001), 10651081. 
30. H. S. Wilf and D. Zeilberger. "Rational function certification of hypergeometric multiintegral/sum/“" q"identities," Invent. Math., 108 (1992), 575-633.

31. D. Zeilberger. "A fast algorithm for proving termininating hypergeometric identities," Discrete Math., 80 (1990), 207-211. 